\title{
Scattering Functions of Semidilute Solutions of Polymers in a Good Solvent
}

\author{
JAN SKOV PEDERSEN, ${ }^{1}$ PETER SCHURTENBERGER ${ }^{2}$ \\ ${ }^{1}$ Department of Chemistry and iNANO Interdisciplinary Nanoscience Center, University of Aarhus, Langelandsgade 140, \\ DK-8000 Aarhus C, Denmark \\ ${ }^{2}$ Department of Physics, University of Fribourg, Pérolles, CH-1700 Fribourg, Switzerland
}

Received 14 January 2004; accepted 6 April 2004

DOI: 10.1002/polb.20173

\begin{abstract}
Expressions for analyzing small-angle scattering data from semidilute solutions of polymers in a good solvent over a broad range of scattering vectors are examined. Three different scattering function expressions are derived from Monte Carlo simulations. The expressions are similar to those of polymer reference interaction site models, with a scattering-vector-dependent direct correlation function. In the most advanced model, the screening of excluded-volume interactions beyond the overlap concentration is taken into account. Two simpler expressions, in which the screening of excluded-volume interactions is not included, are also applied. The three models are tested against small-angle neutron scattering (SANS) experiments on polystyrene in deuterated toluene for a broad range of molar masses and concentrations over a wide range of scattering vectors. For each model, simultaneous fits to all the measured scattering data are performed. The most advanced model excellently reproduces the SANS data over the full range of the parameters. The two simpler models fit the data almost equally well. On the basis of an extensive study, an optimal fitting strategy can be recommended for experimentalists, who want to analyze small-angle scattering data from polymers at any concentration. For data sets that do not contain data on the single-chain scattering function, the simpler model is recommended; it uses a direct correlation function equal to the form factor of an infinitely thin rod, which is independent of the concentration and molar mass. (C) 2004 Wiley Periodicals, Inc. J Polym Sci Part B: Polym Phys 42: 3081-3094, 2004
\end{abstract}

Keywords: polystyrene; solution properties; neutron scattering; theory; Monte Carlo simulation

\section{INTRODUCTION}

The properties of dilute and semidilute polymer solutions have fascinated scientists for a long time because of the universal behavior that they exhibit, which is independent of the chemical details of the polymer-solvent systems under con-

Correspondence to: J. S. Pedersen (E-mail: jsp@chem. au.dk) sideration. The behavior and properties are mainly determined by the connectivity and flexibility of the polymer chains and by the presence or absence of excluded-volume interactions. Although the solution properties are universal, their detailed and quantitative theoretical description remains a real challenge, and many different approaches have been employed. Among these are scaling theory, ${ }^{1}$ which only provides qualitative insight. Another approach is to use renormalization group theory (RGT), which is able to give a quantitative description. ${ }^{2-4}$ The last 
of the main approaches to be mentioned in this context is the use of simulation techniques. ${ }^{5}$ With this approach, data and results for well-defined models and systems can be generated, and the results can be compared with experimental data or used for testing various theoretical approaches.

The main experimental approach for obtaining structural and thermodynamic results for dilute and semidilute polymer systems is the use of scattering techniques. ${ }^{6}$ These techniques give direct access to the ensemble averages of the characteristic length scales and to the interparticle interactions through either the virial coefficients or the osmotic compressibility. For semidilute polymer solutions, the scattering function $[S(q)]$ at a zero scattering vector modulus $(q=0)$ is inversely proportional to the osmotic compressibility $[d \Pi(c) / d c]$ of the system, whereas the correlation length $\xi$ can be obtained from the initial $q$ dependence of $S(q) \approx S(0)\left[1-\xi^{2} q^{2}\right]$, where $S(0)$ is the forward scattering. At very low concentrations, $\xi$ is given by the size of a single chain as $\xi=R_{g} / \sqrt{3}$, where $R_{\mathrm{g}}$ is the radius of gyration of the polymer chains. $S(0)$ is related to the second virial coefficient $\left(A_{2}\right)$ by $S(0) \approx 1-2 c M A_{2}$, where $c$ is the polymer concentration and $M$ is the polymer molar mass. At higher concentrations, above the overlap concentration of the chains $\left(c^{*}\right), \xi$ is the correlation length of the transient network of interpenetrating chains. Both $d \Pi(c) / d c$ and $\xi$ can be obtained by light scattering (LS), small-angle neutron scattering (SANS), or small-angle X-ray scattering measurements, depending on the molar weight of the polymer and the concentration. In this study, we go beyond an analysis of the low- $q$ data and examine the full dependence of $S(q)$ on $q$. The scattering function expressions are derived from Monte Carlo simulations. ${ }^{7-9}$ They are related to the expressions from the randomphase approximation (RPA) $)^{10-12}$ and the more advanced polymer reference interaction site model (PRISM). ${ }^{13}$ The various model expressions are tested against SANS data from polystyrene (PS) in the good solvent toluene.

There are numerous reports in the literature on previous studies of PS in good solvents. In this context, we only summarize the publications that are most important with respect to the work presented here. PS in good and $\theta$ solvents was investigated by Yamamato et al., ${ }^{14}$ Fukuda et al., ${ }^{15}$ and Miyaki et al. ${ }^{16}$ with LS. They provided scaling reactions for $R_{\mathrm{g}}$ and $A_{2}$, which in some studies have also been related to the interpenetration function. Huber et al. ${ }^{17}$ employed LS in combination with SANS for the same purpose. Semidilute solutions and the associated scaling behavior with an emphasis on $\xi$ were investigated by Cotton et al., ${ }^{18}$ Daoud et al., ${ }^{10}$ Wiltzius et al., ${ }^{19}$ Koberstein et al. ${ }^{20}$ and Brown et al. ${ }^{21}$ Most of these studies were done with SANS. Brown et al. further measured relatively high-concentration systems and found a crossover into a dense regime where the scaling breaks down. Daoud et al. ${ }^{10}$ and King et al. ${ }^{22}$ established with SANS and labeling techniques and with a determination of $R_{\mathrm{g}}$ as a function of the concentration the screening of excluded-volume effects in the semidilute regime, as predicted by Flory. ${ }^{23}$ Finally, Brown and Nicolai ${ }^{24}$ in 1990 have published an extensive review on scattering studies of semidilute solutions of PS.

The expressions for the scattering functions used in this work are derived from Monte Carlo simulations. In the past, simulation techniques have been widely used for supporting the results from scattering experiments. ${ }^{5}$ The simulation techniques are ideally suited for systems with many degrees of freedom to obtain statistical averages of various parameters and scattering functions. There have been attempts to do full atomistic simulations on polymer systems ${ }^{25}$ with the aim of describing the properties down to the molecular and atomic levels. However, such simulations are not feasible for semidilute solutions of macromolecules because of the gigantic number of atoms and interactions that have to be considered. More useful are mesoscale models, such as the united-atom model for polyethylene,${ }^{26}$ which takes chain rigidity and the torsion angle potential into account. The model uses effective interactions without specifically considering the solvent molecules. Such models allow single-chain and multiple-chain systems to be investigated. To reproduce or describe typical small-angle scatting data, such relatively realistic and detailed models are required. The models have to describe semiflexibility and excluded-volume interactions correctly and should allow the finite cross section of the chains to be taken into account when the scattering functions are calculated for comparison with the experimental data. We have previously shown that a mesoscale model ${ }^{7-9}$ provides a good description of the properties of PS with almost atomic resolution. The model consists of semiflexible chains ${ }^{27}$ with excluded-volume interactions generated by hard spheres placed along the chain. It is somewhat more coarse-grained than the united atom because it considers neither the torsion potentials nor the phenyl side groups of PS. 
However, it is simple enough to allow quite large systems to be investigated.

The model was originally developed for studying wormlike micelles, ${ }^{28,29}$ and most of the applications published in the literature are related to this type of system. The model has also been used in various modified forms for studying electrostatic interactions in charged wormlike micelles, so-called equilibrium polyelectrolyte chains. ${ }^{29-33}$

A brief account of some of the results in this article has been published previously. ${ }^{9}$ In the previous article, the expressions for the scattering functions derived from a Monte Carlo simulations are not given, and only one model is applied for fitting the concentration series from only one molecular weight of PS. In this article, we describe the details of three models of different complexities, which are similarly derived from Monte Carlo simulations. The full expressions are given because this will allow other investigators to use them in the future. The applicability of the three models is investigated using them for a large SANS data set recorded for six different molar masses of PS. In contrast to the previous publication, this allows the fitting results to be investigated as a function of the molar mass, and this makes it possible to obtain a much more detailed cross-check of the consistency of the models and the data.

The article is organized as follows. In the next section, we describe the simulations and the model. In the third section, the expressions for the scattering function of the models are given. The experimental conditions are summarized in the fourth section. The results from a scaling analysis, a virial analysis, and the models of $S(q)$ are described in the fifth section, whereas a discussion and a summary of the work are given in the final section.

\section{SIMULATIONS}

The model expressions presented in the next section are based on an extensive Monte Carlo simulation study of the scattering functions of semiflexible chains interacting with a hard-sphere potential. The polymer model has a fixed valence angle $(\theta)$ and free rotation around the bonds..$^{7,9,27}$ The fixed valence angle $\theta$ gives rise to the semiflexibility. Hard spheres are placed along the chain to simulate the excluded volume of the polymer. In the simulations, the chains have six-toeight spheres per statistical segment (Kuhn) length $(b)$, and the full multiple-chain system typ- ically contains $10000-30000$ spheres of radius $R / b=0.1$. The contour length of a chain is $L$ $=N l_{0}$, where $N+1$ is the number of points/ spheres along the chain and $l_{0}$ is the distance between them. $b$ is related to $\theta$ by $b=l_{0}(1+\cos$ $\theta) /(1-\cos \theta)$. A box with periodic boundary conditions has been used to reduce the influence of the finite size of the system.

In the dilute region, pivot moves ${ }^{7,9,34}$ have been used for moving the chains, and the longest part of a chain has been pivoted as this gives rise to movements of the center of the mass. The pivot moves are very effective at low concentrations but become quite inefficient at higher concentrations. Therefore, reptation (cut-and-paste) moves have been used at higher concentrations. The total number of moves in a simulation is typically $10^{6}-10^{7}$.

The scattering functions have been calculated as described in refs. 37 and 38 and sampled together with the square of $R_{\mathrm{g}}$. Both the singlechain $\left[P_{\mathrm{sc}}(q)\right]$ and full-system $[S(q)]$ scattering functions have been sampled. Standard errors for $S(q), P_{\mathrm{sc}}(q)$, and $R_{\mathrm{g}}$ have been determined by block analysis.

Simulations have been performed as a function of the chain length and concentration. The chain lengths are $L / b=3.2,10.88,30,90$, and 270. For PS, $b$ is about $20 \AA{ }^{39,40}$ the projected contour length of a monomer $\left(l_{\mathrm{PS}}\right)$ is about $2.50 \AA$, and the molar mass per monomer $\left(M_{\mathrm{PS}}\right)$ is 104 . From these parameters, the corresponding molar mass can be estimated as $M=M_{\mathrm{PS}}(L / b) b / l_{\mathrm{PS}}$. The $L$ values thus correspond to molar masses of about $2700,9100,25,000,75,000$ and 225,000. For each value of $L / b$, the concentration has been varied from very dilute concentrations well below $c^{*}$ up to concentrations with volume fractions of $\eta$ $=0.1-0.25 .^{41}$ For PS, the highest volume fractions are close to the melt density. ${ }^{42}$

\section{MODELING}

In this section, we present the model expressions that are to be tested by being fitted to an extensive data set from PS in toluene. A very detailed comparison between the simulations and experiments is possible through a comparison of the full $q$ dependence of $S(q)$. As the simulations and experiments have not been performed with exactly the same concentrations and contour lengths, a comparison requires an interpolation of the simulated functions. The derivation of such expres- 
sions also allows them to be used by others in the future. $^{43}$

\section{Full Model Based on Simulations}

The agreement of the sampled scattering functions with the classical RPA results ${ }^{10-12,35}$

$$
S_{\mathrm{RPA}}(q)=\frac{P_{\mathrm{sc}}(q)}{1+\beta P_{\mathrm{sc}}(q)}
$$

was initially investigated. In eq $1, P_{\mathrm{sc}}(q)$ is the single-chain form factor. $\beta$ is related to the strength of the interaction potential; however, to obtain agreement for $S(0), \beta$ must fulfill $\beta$ $=S(0)^{-1}-1$, and we can use the RGT results for estimating $\beta . P_{\mathrm{sc}}(q)$ varies with the concentration because of the screening of excluded-volume effects and the corresponding variation of $R_{\mathrm{g}}$ at high concentrations.

The function $S_{\mathrm{RPA}}(q)$ is not able to describe the simulation data, and large deviations are found, particularly for large concentrations at high $q$. An alternative approach is to use the polymer version of the integral equation theory of liquids ${ }^{44}$ (PRISM), which in the equivalent site approximation gives ${ }^{13}$

$$
S_{\mathrm{PRISM}}(q)=\frac{P_{\mathrm{sc}}(q)}{1+\beta c(q) P_{\mathrm{sc}}(q)}
$$

where $c(q)$ is the normalized Fourier transform of the direct correlation function for the spheres on the chains.

The various dependencies and functions entering eq 2 have been analyzed in detail, and a series of empirical parameterizations have been obtained. These are summarized in the following.

The expression for $P_{\mathrm{sc}}(q)$ is based on the parameterization (method 1) of Pedersen et al. ${ }^{7}$ However, it is also necessary to included the screening of the excluded-volume interactions at high concentrations. $R_{\mathrm{g}}$ at low concentrations is

$$
R_{\mathrm{g}}^{2}=R_{\mathrm{g}, 0}^{2} \alpha(L / b)^{2}
$$

where $R_{\mathrm{g}, 0}$ is the radius of gyration of ideal semiflexible chains: ${ }^{45}$

$$
R_{\mathrm{g}, 0}^{2}=\frac{L b}{6}-\frac{b^{2}}{4}+\frac{1}{4 L / b}-\frac{1}{8(L / b)^{2}}[1-\exp (-2 L / b)]
$$

$\alpha(x)$ is the expansion coefficient originating from excluded-volume interactions:

$$
\alpha(x)^{2}=\left[1+\left(x / x_{2}\right)^{2}+\left(x / x_{3}\right)^{3}\right]^{\epsilon / 3}
$$

The values $x_{2}=3.12, x_{3}=8.67$, and $\epsilon=0.170$ have been determined from single-chain simulations. $^{7}$

The screening of excluded-volume interactions at high concentrations results in a rescaling of $R_{\mathrm{g}}$ :

$$
\tilde{R}_{\mathrm{g}}=S_{R_{\mathrm{g}}} R_{\mathrm{g}}
$$

where $S_{R_{\mathrm{g}}}$ is a concentration-dependent scale factor less than or equal to unity, so that $\tilde{R}_{\mathrm{g}}$ is always smaller than or equal to $R_{\mathrm{g}}$. The screening of excluded-volume interactions at high concentrations depends on the reduced concentration $(X)$, which is approximately $c / c^{*} . X$ is chosen in agreement with the RGT calculation: ${ }^{2}$

$$
X=\frac{16}{9} A_{2} c M
$$

where $c$ is the concentration $(\mathrm{g} / \mathrm{mL})$. The following empirical expressions can describe $S_{R_{\mathrm{g}}}$ of $R_{\mathrm{g}}$ :

$$
S_{R_{\mathrm{g}}}=\frac{\left[\left(C_{R_{\mathrm{g}}}\right)^{\alpha_{1}}+\left(B_{R_{\mathrm{g}}}\right)^{\alpha_{1}}\right]^{1 / \alpha_{1}}}{\left[1+\left(C_{R_{\mathrm{g}}}\right)^{\alpha_{1}}\right]^{1 / \alpha_{1}}}
$$

where

$$
C_{R_{\mathrm{g}}}=\frac{\alpha(x)}{[1-\alpha(x)]^{\beta_{1}}}
$$

and

$$
B_{R_{\mathrm{g}}}=\left[1+\left(X a_{1}\right)^{\gamma_{1}}\right]^{\gamma_{2}}
$$

In these expressions, the following parameters are determined via fitting to the simulated scattering functions: $\alpha_{1}=38.72, \beta_{1}=0.01381, \gamma_{1}$ $=0.995$, and $\gamma_{2}=-0.1156$. The parameter $a_{1}$ relates the reduced polymer concentration calculated from the polymer concentration of the samples in the experiment to the reduced concentration calculated volume fraction in the simulations. Therefore, we have taken it as a variable parameter and optimized it during the fitting to the scattering data. In this way, it is possible in the fit to have the screening of the excluded-volume interactions occurring correctly with respect 
to the reduced concentration for the polymer system.

$P_{\mathrm{sc}}(q)$ has been sampled in the simulations, and the full $q$ dependence can be described by an empirical expression similar to the one used in ref. 8:

$$
\begin{aligned}
P_{\mathrm{sc}}(q)=\left[P_{\text {chain }}(q)(1\right. & -\chi(q, L, b)) \\
& \left.+P_{\text {rod }} \chi(q, L, b)\right] \Gamma(q, L, b)
\end{aligned}
$$

where $P_{\text {chain }}(q)$ is the scattering function of flexible chains, $P_{\text {rod }}(q)$ is the scattering function of an infinitely thin rod, and $\chi(q, L, b)$ and $\gamma(q, L, b)$ are crossover functions. Expressions for these two functions can be found in ref. 8. In the highconcentration region, in which the excluded-volume interactions are strongly screened, $P_{\text {chain }}(q)$ is steeper than the Debye function for Gaussian chains (as discussed later) in the region in which the function crosses over to power-law behavior. Therefore, the following expression has been used:

$$
P_{\text {chain }}(q)=\operatorname{MAX}\left\{P_{\text {Amp }}(q), P_{\text {Gauss }}(q), P_{\text {exv }}(q)\right\}
$$

In these equations, $P_{\mathrm{Amp}}(q)$ is equal to $(1-\exp$ $Y) / Y$, where $Y=2 \tilde{R}_{\mathrm{g}}^{2} q^{2} / 3, P_{\text {Gauss }}(q)$ is equal to $2(\exp Z-1+Z] / Z^{2}$, where $Z=\tilde{R}_{\mathrm{g}}^{2} q^{2}$ is the scattering function of Gaussian chains, and $P_{\text {exv }}(q)$ is the scattering function of self-avoiding chains with $R_{\mathrm{g}}$.

$c(q)$, which also has a concentration dependence, can be described by the rod scattering function:

$$
c(q)=P_{\text {rod }}\left(q, R_{\mathrm{g}}^{c(q)}\right)
$$

The $q$ dependence is given by the following parameter:

$$
\begin{array}{r}
R_{\mathrm{g}}^{c(q)}=\left[\delta_{1} \tilde{R}_{\mathrm{g}}^{\delta_{2}} \exp \left(-\left(X_{2}+3\right)^{2} / 2\right)+\delta_{3}\right] b \\
\text { for } X_{2} \geq-3,
\end{array}
$$

$$
R_{\mathrm{g}}^{c(q)}=\left[\delta_{1} \tilde{R}_{\mathrm{g}}^{\delta_{2}}+\delta_{3}\right] b \text { elsewhere }
$$

In these equations, $X_{2}$ is equal to $\log \left(a_{2} c\right)$, where $a_{2}$ is again a parameter that relates the polymer concentration in the experiment to the volume fraction in the simulation. It has been taken as a fitting parameter and obtained via fitting to the SANS data. The function eq 14 approaches the (length-independent) hard-sphere fluid value at a high concentration, and the parameter $a_{2}$ controls the approach. The parameters are determined from the fitting of the simulation results: $\delta_{1}$ $=0.2368, \delta_{2}=0.7340$, and $\delta_{3}=0.155$.

The parameter $\beta$ in eq 2 can be described by a function based on a result from RGT. ${ }^{2,3}$ We use

$$
\begin{aligned}
\beta=\frac{1}{8}\left[9 X_{3}-2+\right. & \left.2 \log \left(1+X_{3}\right) / X_{3}\right] \exp \left[1 / X_{3}\right. \\
& \left.-\left(1-1 / X_{3}^{2}\right) \log \left[1+X_{3}\right] / \epsilon_{1}\right] \times \\
& \times \exp \left[-\left(a_{3} c \varepsilon_{2}\right)^{\epsilon 3}\right]
\end{aligned}
$$

where the first part of the expression is a modified version of the RGT, in which the scaling from the simulations is included. The last part of the expression describes the crossover to the high-concentration regime, in which the scaling breaks down. In the expression, $X_{3}=X \epsilon_{4}$ is a reduced concentration parameter. ${ }^{46} a_{3}$ is the last parameter relating the polymer concentration in the experiments to the volume fraction in the simulations. ${ }^{47}$ It has been taken as a fitting parameter and optimized via fitting to the SANS data. The rest of the parameters have been determined via fitting to the simulation results: $\epsilon_{1}=2.565, \epsilon_{2}$ $=3.200, \epsilon_{3}=1.050$, and $\epsilon_{4}=0.9087 . \epsilon_{1}$ gives the scaling $S(0) \propto c^{-1-1 / 2.565}=c^{-1.390}$ for values of $c$ large in comparison with $c^{*}$.

The differential scattering cross section normalized by the concentration is given by

$$
\frac{d \sigma}{d \Omega}(q)=M \Delta \rho_{m}^{2} S_{\mathrm{PRISM}}(q) S_{\mathrm{xs}}(x)+B
$$

where $\Delta \rho_{\mathrm{m}}$ is the excess scattering length density of PS in toluene per unit of mass of the polymer and $B$ is a constant accounting for residual incoherent background. The cross-section scattering function is

$$
S_{\mathrm{xs}}(x)=\left[\frac{2 B_{1}(q R)}{q R}\right]^{2}
$$

where $B_{1}(x)$ is a first-order Bessel function and $R$ is the cross-section radius.

\section{Simpler Models}

The parameterizations of the screening of excludedvolume interactions in the single-chain scattering functions lead to rather complicated expressions. In experiments, the screening cannot be observed di- 
rectly unless special labeling schemes are applied. The RPA/PRISM expression can be used to explain this. In full-system measurements at finite concentrations, the nominator and denominator of the RPA/PRISM expression are approximately the same at low $q$, at which the screening effects occur in the single-chain scattering function. Thus, the screening cannot be observed directly. We have, therefore, attempted to use simply the single-chain scattering functions of excluded-volume chains in the PRISM expression. One further complicated component of the full model is the concentration and contour length dependence of $c(q)$. We have tested two alternative simpler approximations for this function. The first model (I) is a simple correlation hole model, which uses the following expression for $c(q):^{48}$

$$
c(q)=\frac{3\left[\sin \left(q 2 R_{\mathrm{HS}}\right)-q 2 R_{\mathrm{HS}} \cos \left(q 2 R_{\mathrm{HS}}\right)\right]}{\left(q 2 R_{\mathrm{HS}}\right)^{3}}
$$

where $R_{\mathrm{HS}}$ is an effective hard-sphere radius. For $\beta$, which is directly related to the forward scattering by $S(0)=1 /(1+\beta)$, we use eq 15 .

The second model (II) uses a direct correlation function equal to the form factor of an infinitely thin rod as done in the full model, but it takes $R_{\mathrm{g}}$, related to the interaction distance, to be independent of the concentration and chain length. For $\beta$, we again use eq 15 .

\section{EXPERIMENTAL}

Full-system scattering functions have been determined experimentally by SANS measurements on PS in a good solvent deuterated toluene ( $d$-toluene). The polymers were standards from various sources with low polydispersity indices $(<1.1)$. The $M$ values were $3000,13,000,28,000,68,000$, 120,000 , and $350,000 \mathrm{Da} . d$-Toluene with a degree of deuteration higher than 99\% was acquired from Sigma. Samples with concentrations of 5, 10, 25 , and 50 wt $\%$ were prepared gravimetrically and left for a week. The samples with $50 \mathrm{wt} \%$ of the 120,000 and 350,000 Da polymers were too viscous to be transferred to the quartz cells used for the SANS measurements, and they were, therefore, not measured. Samples with lower concentrations (for the 350,000 Da polymer down to 0.05 wt \%) were prepared by dilution.

The measurements were performed at the SANS instrument at Ris $\emptyset$ National Laboratory ${ }^{36}$ and covered $q=0.004-0.5 \AA^{-1}$ with a wave- length resolution of $18 \%$ (full width at half-maximum). The sample-detector distances and neutron wavelengths were, for the three settings used, $1.0 \mathrm{~m}$ and $2.8 \AA, 3.0 \mathrm{~m}$ and $4.5 \AA$, and $6.0 \mathrm{~m}$ and $10.0 \AA$. The samples were measured in standard Hellma quartz cells with a path length of 1-10 mm, which depended on the concentration $(0.0005-0.56 \mathrm{~g} / \mathrm{mL})$. The SANS data were converted to an absolute scale with a 1-mm-thick water sample as a secondary standard. In connection with the use of water for absolute calibration, an empirical wavelength-dependent factor was used. This factor takes into account multiple scattering, neutron thermalization in the water, and the wavelength dependence of the detector efficiency ${ }^{49}$ For the Ris $\emptyset$ SANS, the correction factor of the detector was not determined experimentally, and we used a factor based on literature values. This led to significant uncertainty on the absolute scale of the data. However, as this is just a constant and is the same for all data, it has no influence on the conclusions in this article.

We corrected minor concentration errors introduced during the dilutions by scaling the data at high $q$ beyond the cross-section Guinier region. An incoherent background of $0.287 \mathrm{~cm}^{-1} \mathrm{~mL} / \mathrm{g}$ from PS was subtracted from all data sets.

\section{RESULTS}

The SANS data are shown in Figure 1. The data for the different molar masses show qualitatively similar behaviors. At the lowest concentrations, the influence of the concentration is modest, and the data approach the single-chain scattering function. It behaves as $q^{-1.67}$ at intermediate $q$ values, and at higher $q$ values, at which it is expected to cross over to $q^{-1}$, the cross-section scattering function sets in and results in a strong decrease in the intensity. 8,40

\section{Scaling Analysis}

To establish the scaling behavior of $S(0)$ and $\xi$, we have analyzed the simulated and measured fullsystem scattering functions with the same approach. This also allows a first comparison between the simulations and experiments. $S(0)$ and $\xi$ have been determined by the fitting of the Debye expression for Gaussian chains ${ }^{50}$

$$
S(q)=S(0) P_{\text {Gauss }}(x)=S(0) \frac{2[\exp (-x)-1+x]}{x^{2}}
$$



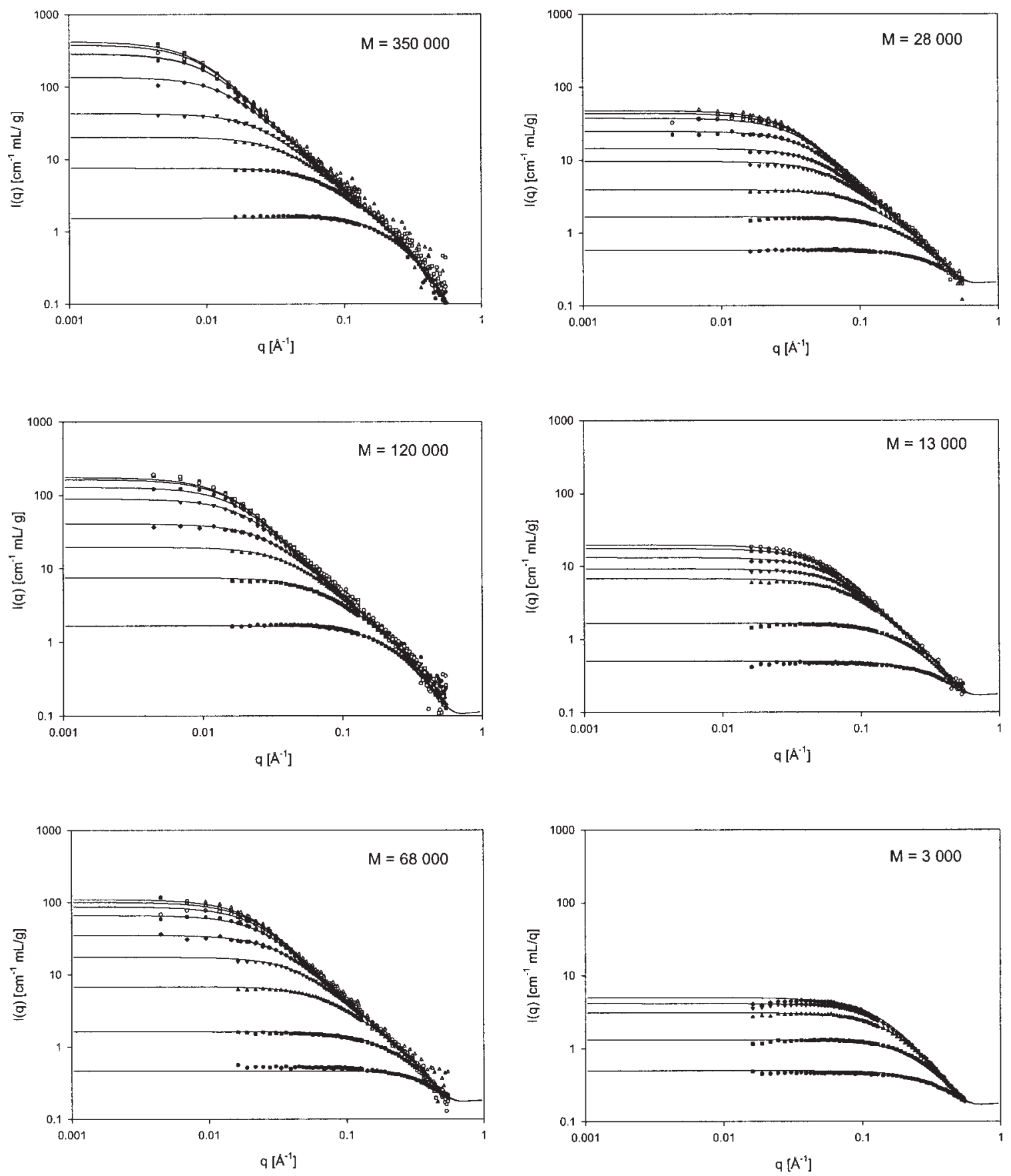

Figure 1. $S(q)$ for PS samples of different molecular weights. For $M=350,000$, the $c$ values are, from bottom to top, 0.271, 0.102, 0.0539, 0.0270, 0.0103, 0.00329, 0.00251, and $0.00102 \mathrm{~g} / \mathrm{mL}$. For $M=120,000$, the $c$ values are, from bottom to top, $0.269,0.108$, $0.0539,0.0283,0.0109,0.00523,0.00206$, and $0.000908 \mathrm{~g} / \mathrm{mL}$. For $M=68,000$, the $c$ values are, from bottom to top, $0.562,0.279,0.114,0.0552,0.0279,0.0111,0.00548$, 0.00279 , and $0.00119 \mathrm{~g} / \mathrm{mL}$. For $M=28,000$, the $c$ values are, from bottom to top, 0.517 , $0.279,0.162,0.0808,0.0542,0.0269,0.0107,0.00536$, and $0.00267 \mathrm{~g} / \mathrm{mL}$. For $M$ $=13,000$, the $c$ values are, from bottom to top, 0.557, 0.277, 0.0897, 0.0545, 0.0276, 0.0112 , and $0.00505 \mathrm{~g} / \mathrm{mL}$. For $M=3000$, the $c$ values are, from bottom to top, 0.541 , $0.271,0.103,0.0541$, and $0.0253 \mathrm{~g} / \mathrm{mL}$. The curves are fit using a PRISM-type parameterization of Monte Carlo simulation results. 

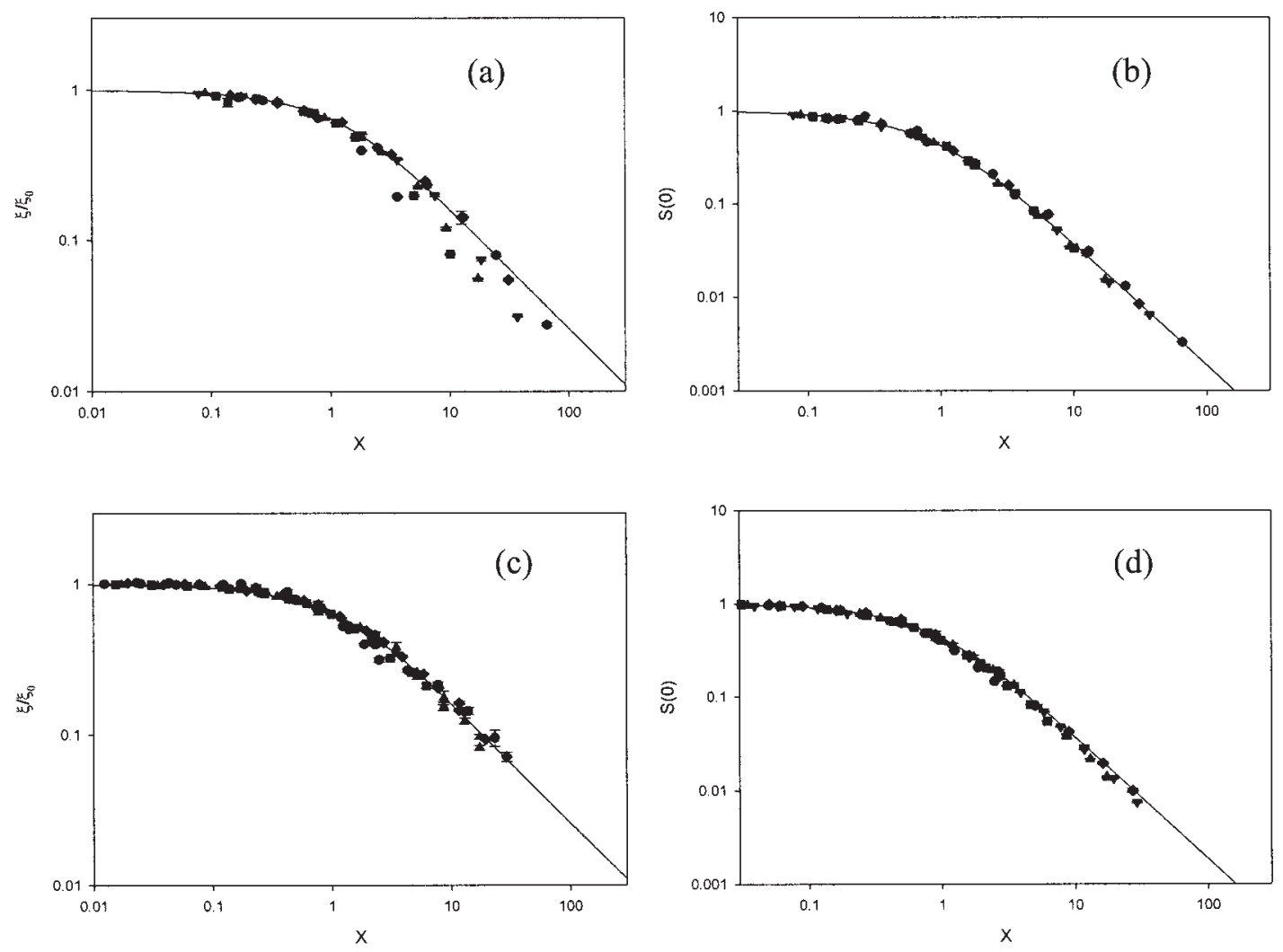

Figure 2. Parameters $\xi$ and $S(0)$ as functions of $X$ : (a) $\xi$ for SANS of PS for $M=(\bullet)$

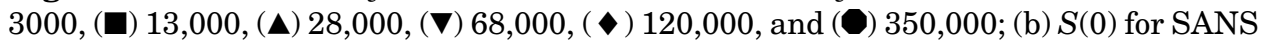

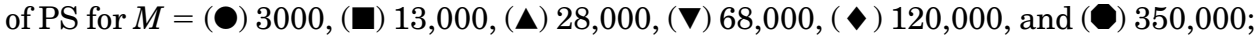
(c) $\xi$ for the simulations for $L / b=(\bullet) 3.2$, ( $\mathbf{\square}) 10.88,(\boldsymbol{\Delta}) 30,(\boldsymbol{\nabla})$ 90, and ( ) 270; and (d)

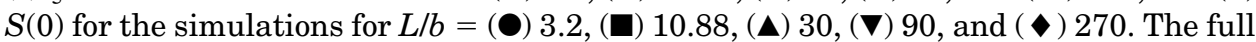
curves are the RGT results of Schäfer. ${ }^{4,57}$

to the low- $q$ portion of the data. This function describes the data to higher $q$ values than a standard Onstein-Zernike (OZ) expression: $S(q)$ $=S(0) /\left(1+\xi^{2} q^{2}\right)$. In the Debye function, $x$ is equal to $R_{\mathrm{g}}^{2} q^{2}$, but to obtain a correlation length in agreement with the usual definition from the Lorentzian, we set $x$ equal to $3 \xi^{2} q^{2}$. The Debye function has been fitted to the data with $q \xi<1$. The OZ expression corresponds to an exponentially decaying correlation function and has traditionally been used because it is possible to derive $S(0)$ and $\xi$ from a plot of $1 / S(q)$ versus $q^{2}$ by a straight-line approximation. However, the Debye expression takes into account the connectivity and flexibility of the chain (although in an approximation without excluded-volume), and so it gives a good fit to higher $q$ values and is consequently easier to apply during fitting. The applicability of the expression to lower $q$ values is important for SANS data, which are often difficult to obtain at low $q$ values. The OZ and Debye expressions give the same results when the fits are limited to very low values of $q$.
To investigate the scaling, it is appropriate to plot the parameters as a function of $X=\alpha c / c^{*}=$ $(16 / 9) c A_{2} M$, where $\alpha$ is a constant of order unity. ${ }^{2}$ The scale factors for the concentration axis in the SANS and simulation data have been determined through the fitting of $S(0)=1 /[1+\beta]$ with $\beta$ from eq 15 to the data. These scale factors from $S(0)$ have also been used for the $\xi$ data.

The simulation and SANS data for $S(0)$ and $\xi$, shown in Figure 2, are in very good agreement and do display scaling. The results also agree well with the RGT results of Schäfer. ${ }^{4}$ The results of Schäfer have been scaled so that the definition $X$ $=(16 / 9) c A_{2} M$ from ref. 2 is valid. In the plot, some deviations from scaling can be observed at high concentrations as the dense region is entered. ${ }^{21}$

\section{Virial Analysis}

Before analyzing the full sets of data, we have checked the consistency of the data sets for each of the molar masses by making a Zimm/RPA-type analysis of only the three lowest concentrations in 
the low- $q$ region. The following expression has been used:

$$
\frac{d \sigma}{d \Omega}(q)=\text { Scale } \frac{P_{\text {Gauss }}\left(q R_{\mathrm{g}}\right)}{1+c 2 M A_{2} P_{\text {Gauss }}\left(q R_{\mathrm{g}}\right)}
$$

where $S_{\text {Gauss }}\left(q R_{\mathrm{g}}\right)$ is the scattering function of Gaussian chains with $R_{\mathrm{g}}$. Scale, $A_{2}$, and $R_{\mathrm{g}}$ are fitting parameters. The Debye expression is here again used because of its more realistic inclusion of correlations within the polymer system in comparison with the usual Zimm expression, which is similar to the $\mathrm{OZ}$ expression.

The scale factor fulfills Scale $=M \Delta \rho_{\mathrm{m}}^{2}$ and should thus scale linearly with $M$. A double logarithmic plot is shown in Figure 3(a). The curve is a fit with a straight line. The good agreement confirms the expected linear dependence, which gives confidence in the consistency of the data sets. The scale factor follows: Scale $=(0.001812$ $\pm 0.000091) M \mathrm{~cm}^{-1} \mathrm{~g} / \mathrm{mL}$.

A value for the scale factor can be calculated from the partial specific densities and the neutron scattering lengths. For a PS density of $1.09 \mathrm{~g} / \mathrm{cm}^{3}$ ${ }^{51}$ and a number density of $d$-toluene molecules calculated from the density of protonated toluene of $0.867 \mathrm{~g} / \mathrm{cm}^{3}$ and the molar mass of protonated toluene, we can obtain a contrast per gram of PS of $\Delta \rho_{\mathrm{m}}=3.84 \times 10^{10} \mathrm{~cm} / \mathrm{g}$. When $M$ of PS is used, the scale factor is given by Scale $=M \Delta \rho_{\mathrm{m}}^{2} / N_{\mathrm{A}}$ $=0.00246 \mathrm{M} \mathrm{cm}^{-1} \mathrm{~g} / \mathrm{mL}$, where $N_{\mathrm{A}}$ is Avogadro's number. There is a quite large deviation of about $30 \%$ between the observed and calculated scale factors. We believe that this is associated with a systematic error from the conversion of the data to the absolute scale. Huber et al. ${ }^{17}$ in their SANS and LS study found a disagreement in the masses derived from the two types of experiments and reported that the masses from SANS were 5-20\% lower than the corresponding masses from LS. Therefore, we have to consider whether the discrepancy could be due to a common error in the SANS approach. Huber et al. ascribed the discrepancy to an inaccuracy in the determination of the contrast factor. In this study, we have used the measured apparent specific volume of PS in toluene ${ }^{51}$ in our calculation of the contrast factors, and we have also checked the value with a Paar DMA5000 density meter. ${ }^{52}$ Therefore, our contrast factor should be correct with respect to this factor. We note that Huber et al. did not quote the partial specific density that they used in their calculation of the contrast factor. The only alternative explanation that we can suggest is the
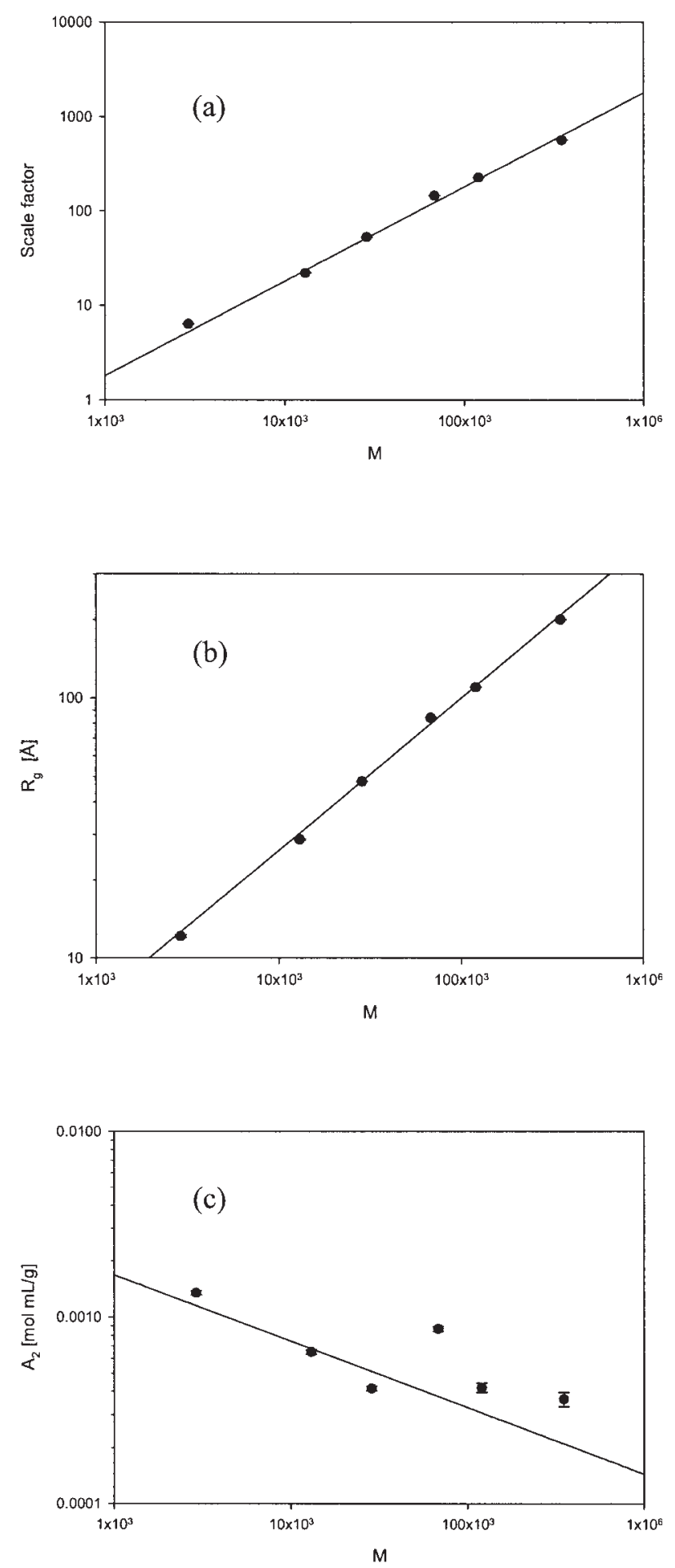

Figure 3. Results from Zimm-type fits of the low- $q$ portion of the data: (a) a scale factor with a linear fit passing through $(0,0)$, (b) $R_{\mathrm{g}}$ with a power-law fit, and (c) $A_{2}$ with a power-law fit.

possibility that inelastic effects influence the scattering data and give a general reduction of the measured intensity. 
Table 1. Scaling Relations for $R_{\mathrm{g}}$

\begin{tabular}{|c|c|}
\hline Relation & Reference \\
\hline$R_{\mathrm{g}}=0.143 M^{0.580} \AA$ & $\begin{array}{l}\text { Yamamoto } \\
\text { et al. }{ }^{14}\end{array}$ \\
\hline$R_{\mathrm{g}}=0.1212 M^{0.575} \AA$ & $\begin{array}{l}\text { Miyaki } \\
\text { et al. }{ }^{16}\end{array}$ \\
\hline$R_{\mathrm{g}}=0.152 M^{0.580} \AA$ & $\begin{array}{l}\text { Fukuda } \\
\text { et al. }{ }^{15}\end{array}$ \\
\hline$R_{\mathrm{g}}=0.125 M^{0.595} \AA$ & Huber et al. ${ }^{17}$ \\
\hline$R_{\mathrm{g}}^{\mathrm{b}}=(0.120 \pm 0.021) M^{0.585 \pm 0.016 \AA}$ & This work \\
\hline
\end{tabular}

$R_{\mathrm{g}}$ is shown in Figure 3(b). It displays a powerlaw behavior:

$$
R_{\mathrm{g}}=(0.120 \pm 0.021) M^{0.585 \pm 0.016} \AA
$$

where $M$ (as in the expressions later for $R_{\mathrm{g}}, L$, and $A_{2}$ ) is measured in grams per mole. The exponent in the equation is in agreement with the excluded-volume value (0.588) found by RGT ${ }^{53}$ and simulations. ${ }^{54}$ The behavior is in good agreement with previous studies ${ }^{14-17}$ of PS in good solvents (see Table 1).

$A_{2}$ is shown in Figure 3(c). The data follow:

$$
A_{2}=(0.020 \pm 0.025) M^{(-0.36 \pm 0.16)} \mathrm{mL} \mathrm{mol} / \mathrm{g}^{2}
$$

It displays a somewhat noisy behavior, and the errors for the prefactor and exponent are quite large; however, the overall behavior is in agreement with the literature ${ }^{15-17,24}$ (see Table 2).

Having now established the consistency of the data, we can continue and test the three models presented in the third section for fitting the full range of scattering vectors and concentrations of the SANS data.

\section{Full Model}

We have fitted simultaneously all data sets for the various concentrations and molar masses. For each molar mass, an overall scale factor, a contour length, a second virial coefficient, and a constant to account for residual incoherent background have been fitted. For all molar weights, a common Kuhn length $b$ and scale factors $a_{1}, a_{2}$, and $a_{3}$ have been fitted. $b$ is determined from the $q$ region in which the data cross over to $q^{-1}$ and in which the cross section scattering function becomes important. The adjustment of the contour length influences only $R_{\mathrm{g}}$ of the chains at infinite dilution and thus allows this parameter to be optimized.

The fits to the data are shown in Figure 1. The fits are excellent with a reduced $\chi^{2}$ value of 7.5. This means that the root-mean-square deviation is only about 2.7 times the standard error for the data from counting statistics. Most of the residuals are found at the highest concentration, and we believe that they are due to an experimental artifact. The data at high concentrations display a slight decrease with smaller scattering vectors. We think that this is due to local saturation effects in the SANS detector due to the relatively high count rates that exist even for a quartz cell with a 1-mm path length. The detector is slightly poorer at the center because of the higher count rates (and possible associated deposits on the wires) that this part of the detector usually experiences for typical samples investigated with the instrument.

$b$ is $17.5 \pm 0.03 \AA$, and the scale factors are $a_{1}$ $=0.736 \pm 0.024, a_{2}=0.06831 \pm 0.00077$, and $a_{3}$ $=0.7075 \pm 0.0014$. We note that $b$ is in good agreement with the literature values. ${ }^{39,40}$ The cross-section radius is $R=5.36 \pm 0.01 \AA$.

$a_{1}$ is a scaling parameter for the reduced concentration and can be expected to be close to unity, which it is. The variable $X_{2}=\log \left(a_{2} c\right)$ in the $c(q)$ function is, when the simulation data are parameterized, $X_{2}=\log \eta$, where $\eta$ is the volume fraction of the spheres on the model chains. Therefore, the fit results in the relation $\eta$ $=0.06831 c$, where $c$ is the concentration $\left(\mathrm{g} / \mathrm{cm}^{3}\right)$.

\begin{tabular}{|c|c|}
\hline Relation & Reference \\
\hline$A_{2}=0.0145 M^{-0.28} \mathrm{~mL} \mathrm{~mol} / \mathrm{g}^{2}$ & Fukuda et al. ${ }^{15}$ \\
\hline$A_{2}=0.0117 M^{-0.262} \mathrm{~mL} \mathrm{~mol} / \mathrm{g}^{2}$ & $\begin{array}{l}\text { Miyaki et al. }{ }^{16} / \text { Brown and } \\
\text { Nicolai }^{24}\end{array}$ \\
\hline$A_{2}=0.00436 M^{-0.203} \mathrm{~mL} \mathrm{~mol} / \mathrm{g}^{2}$ & Huber et al. ${ }^{17}$ \\
\hline$A_{2}=(0.020 \pm 0.025) M^{(-0.36 \pm 0.16)} \mathrm{mL} \mathrm{mol} / \mathrm{g}^{2}$ & This work: Virial analysis \\
\hline$A_{2}=(0.00793 \pm 0.0026) M^{(-0.268 \pm 0.030)} \mathrm{mL} \mathrm{mol} / \mathrm{g}^{2}$ & This work: Modeling \\
\hline
\end{tabular}

Table 2. Scaling Relations for $A_{2}$ 
From the chain density in the melt with a mass density of $c \approx 1.1 \mathrm{~g} / \mathrm{cm}^{3}$, we can estimate ${ }^{42} \eta$ $=0.154$. This results in an expected value of $a_{2}$ of 0.14 , which deviates somewhat from the determined value but has the correct order of magnitude. The parameter $a_{3}$ also relates the volume fraction in simulations to the concentrations in the experiments. We should then expect this parameter to be similar in magnitude to $a_{2}$, but it is much larger. The crossover into the dense region of $S(0)$ is influenced by chemical details, which for PS are related to the quite large phenyl side groups. The influence of the side groups might be smaller on $c(q)$, and so $a_{2}$ is smaller than $a_{3}$.

The scale factors and the $L$ and $A_{2}$ values are plotted in Figure 4. All of these parameters display scaling behavior as a function of $M$. The values of $A_{2}$ are much less noisy than the values determined by the Zimm-type fits. We find the following scaling relations:

$$
\text { Scale }=(0.001627 \pm 0.000091) M \mathrm{~cm}^{-1} \mathrm{~g} / \mathrm{mL}
$$

$$
L=(0.01906 \pm 0.00067) M \AA
$$

$$
\begin{aligned}
A_{2}=(0.00793 & \pm 0.0026) \\
& \times M^{(-0.268 \pm 0.030)} \mathrm{mL} \mathrm{mol} / \mathrm{g}^{2}
\end{aligned}
$$

The scale factor again deviates significantly from the expected value. As before, we believe that the main reason for this is the uncertainty on the absolute scale of the SANS data.

An expression for $L$ can be derived from the expected tetrahedral coordination of the carbon backbone atoms and an expected carbon-carbon distance of $l=1.54 \AA$. Using this value and considering the projection of the $\mathrm{C}-\mathrm{C}$ bond on the contour $\left(\cos 35.3^{\circ}\right)$ we obtain $L=2 l \cos 35.3^{\circ} / M_{\mathrm{PS}}$ $=0.0241 \mathrm{M}$. The prefactor obtained from the fit is thus about $20 \%$ smaller than expected, probably because of a lower expansion of the chains in comparison with the one predicted by the simulation expression (eqs $3-5$ ). In the expression for $R_{\mathrm{g}}$, $L$ enters effectively as $L^{0.586}$, and so the smaller prefactor only results in about $10 \%$ lower values of $R_{\mathrm{g}}$ than expected from the calculated prefactor. The $R_{\mathrm{g}}$ values calculated from the prefactor (eq 24) and $b=17.5 \AA$ are in good agreement with those determined in the virial analysis. The relation for $A_{2}$ is in good agreement with the literature $^{15-17,24}$ (see Table 2).
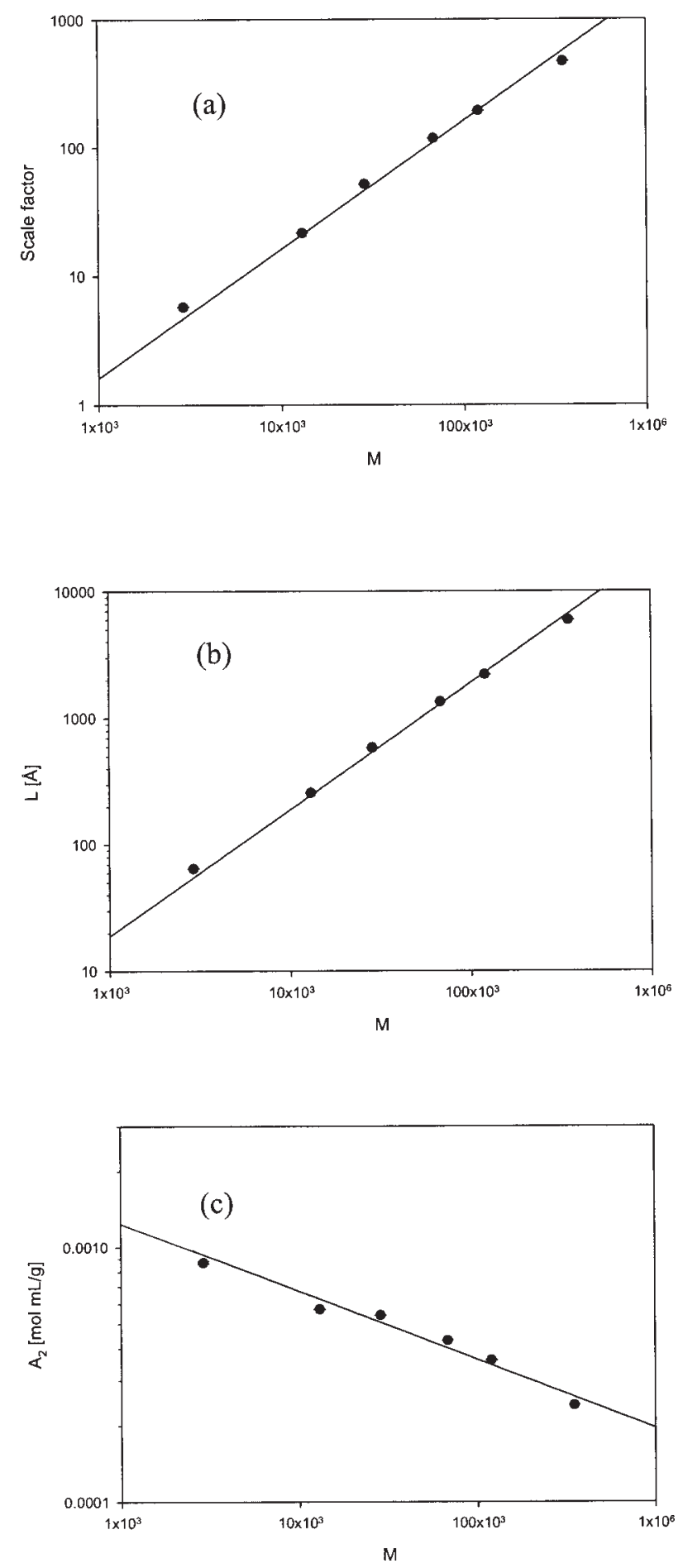

Figure 4. Results from the simultaneous fitting of the full model to the SANS data: (a) a scale factor with a linear fit passing through $(0,0)$, (b) $L$ with a linear fit passing through $(0,0)$, (c) and $A_{2}$ with a power-law fit.

\section{Simpler Model with Hard-Sphere $c(q)$}

The simpler model with a hard-sphere direct correlation function, which is independent of the con- 
tour length and concentration, gives a worse fit than the full model. The agreement with the data of the simpler model is $\chi^{2}=11.5$, corresponding to a root-mean-square deviation of about 3.4 times the standard error for the data from counting statistics. However, this average deviation between the data and model is only $23 \%$ worse than that for the full model, so it is still quite reasonable. In fact, a plot of the fit to the data, as shown in Figure 1, would be almost indistinguishable. We note that most of the additional deviations are for the high-concentration data, for which one is most sensitive to the form of $c(q)$.

$b$ is $16.7 \pm 0.04 \AA$, and $a_{3}$ is $0.6919 \pm 0.0018 . R$ is $5.39 \pm 0.01 \AA$, and $R_{\mathrm{HS}}$ is $4.27 \pm 0.02 \AA$, in good agreement with $R$. The scale factors for the individual molar masses, the contour lengths, and the virial coefficients (not given) are in good agreement with the values for the full model.

\section{Simpler Model with $\operatorname{Rod} c(q)$}

The model with a rod form factor describing the direct correlation function gives a better fit than the model with the hard-sphere direct correlation function. The agreement is $\chi^{2}=9.5$, which is almost as good as that for the full model, which has $\chi^{2}=7.5$. The average deviation between the model and data is thus only about $12 \%$ larger than that for the full model. The better agreement is due to the more correct description of the form of the direct correlation function. This function can be isolated from the simulation with the sampled full-system and single-chain scattering functions, ${ }^{9}$ and it has been found to be in good agreement with a rod form factor. In the simulation, it has been found that the function has a significant concentration and contour length dependence. Despite these effects being neglected in the simpler model, the agreement is very good.

$b$ is $16.7 \pm 0.04 \AA$, and $a_{3}$ is $0.7586 \pm 0.0017 . R$ is $5.43 \pm 0.006 \AA, R_{g}^{c(q)}$ is $6.460 \pm 0.033 \AA$. The values for the scale factors for the various molar masses, contour lengths, and second virial coefficients (not given) are in good agreement with those of the full model.

\section{CONCLUSIONS}

This article focuses on the scattering functions of semidilute solutions of polymers in a good solvent. The aim has been to find model expressions that can describe experimental scattering data over a broad range of scattering vectors for a large range of concentrations and polymer molar masses. The expressions are based on results from an extensive Monte Carlo simulation study in a model with semiflexible chains interacting with a hard-sphere potential. The expression for the scattering function is similar to that of PRISM. To fit the experimental data, we have fully parameterized (and thus also interpolated) the simulated scattering functions. The full parameterization for $S(q)$ takes into account the following effects, which can be observed in the simulation results:

1. The volume fraction dependence of $R_{\mathrm{g}}$ in the calculation of $P_{\mathrm{sc}}(q)$.

2. The deviations from the scaling of $S(0)$ at high volume fractions.

3 . The volume fraction and chain length dependence of $c(q)$, which is described by a rod form factor.

The parameterization reproduces both $P_{\mathrm{sc}}(q)$ and $S(q)$. Two alternative and simpler models in which effects 1 and 3 are not taken into account have also been fitted to the data. In the first model, $c(q)$ is taken as the form factor amplitude of a sphere, and in the second, it is taken as the form factor of an infinitely thin rod.

The models have been tested against SANS data from PS in toluene. In turn, the three models have been fitted to the SANS data through the simultaneous fitting of all the data sets for different molar masses and concentrations. The full model derived from the Monte Carlo simulations provides excellent agreement with the measured data. The determined Kuhn lengths, the contour lengths, and second virial coefficients are in very good agreement with the values and mass dependencies reported in the literature. The simpler model with a hard-sphere direct correlation function provides somewhat poorer, but still reasonable, fits to the data. The model with a direct correlation function equal to the form factor of an infinitely thin rod provides fits to the SANS data of almost the same quality as that of the full model. Because of the simplicity of the model, we recommend using this model for data for which the single-chain scattering functions are not available (which is usually the case). There are also several examples in the literature of applications of the model to semidilute solutions of wormlike micelles, ${ }^{28,29,55,56}$ which basically behave as polymers.

In addition to the modeling, the scaling behavior of $S(0)$ and $\xi$ from the SANS data and the 
simulation data has been checked. These two parameters, both from SANS and simulations, show a universal behavior, with some deviations from scaling when a high-concentration regime is entered. The results are in excellent agreement with the RGT predictions. The low-concentration behavior of the SANS data has been further investigated with a virial analysis. The results from this analysis demonstrate, as expected, excludedvolume scaling of $R_{\mathrm{g}}$ with the molar mass. The results are in good agreement with the literature. The second virial coefficient has also been determined in the virial analysis, but the data are quite noisy. The scaling behavior of the parameters obtained by the full-scale modeling has also been investigated. The scale factor, the contour length, and the second virial coefficient all display the expected behavior with the molar mass of PS.

This work has demonstrated that a mesoscale model with semiflexible chains and an effective excluded-volume interaction is able to describe the observed static properties of PS in toluene. Not only are the osmotic compressibility $[d \Pi(c) /$ $\left.d c \propto S(0)^{-1}\right]$ and $\xi$ reproduced, but the full $q$ dependence of the scattering functions is reproduced as well. The SANS data extend to $q_{\max }$ $=0.5 \AA^{-1}$, which is quite high for a SANS experiment, and the data cover about 2 decades on the length scale. Therefore, the modeling gives a relatively high-resolution interpretation of the data.

This work was financially supported by the Swiss National Science Foundation (grants 20-40339.94 and 2046627.96) and by the Danish Natural Science Research Council. The authors thank Walter Batsberg Pedersen and Kristoffer Almdal for providing the polystyrene used for the small-angle neutron scattering.

\section{REFERENCES AND NOTES}

1. de Gennes, P.-G. Scaling Concepts in Polymer Physics; Cornell University Press: London, 1979.

2. Ohta, T.; Oono, Y. Phys Lett A 1982, 89, 460.

3. Nakanishi, A.; Ohta, T. J Phys A 1985, 18, 127.

4. Schäfer, L. Macromolecules 1984, 17, 1357.

5. Monte Carlo Simulation and Molecular Dynamics Simulation in Polymer Science; Binder, K., Ed.; Oxford University Press: New York, 1995.

6. Higgins, J. S.; Benoit, H. Polymers and Neutron Scattering; Oxford University Press: Oxford, 1994.

7. Pedersen, J. S.; Laso, M.; Schurtenberger, P. Phys Rev E 1996, 54, R5917.

8. Pedersen, J. S.; Schurtenberger, P. Macromolecules 1996, 29, 7602.

9. Pedersen, J. S.; Schurtenberger, P. Europhys Lett 1999, 45, 666 .
10. Daoud, M.; Cotton, J. P.; Farnoux, B.; Jannink, G.; Sarma, G.; Benoit, H.; Duplessix, R.; Picot, C.; de Gennes, P.-G. Macromolecules 1975, 8, 804.

11. Edwards, S. F. Proc Phys Soc London 1966, 88, 265.

12. Benoit, H.; Benmouna, M. Polymer 1984, 25, 1059.

13. Schweizer, K. S.; Curro, J. G. Adv Polym Sci 1994, $116,319$.

14. Yamamoto, A.; Fujii, M.; Tanaka, G.; Yamakawa, H. Polym J 1971, 2, 799.

15. Fukuda, M.; Fukutomi, M.; Kato, Y.; Hashimoto, T. J Polym Sci Polym Phys Ed 1974, 12, 871.

16. Miyaki, Y.; Einaga, Y.; Fujita, H. Macromolecules 1978, 11, 1180.

17. Huber, K.; Bantle, S.; Lutz, P.; Burchard, W. Macromolecules 1985, 18, 1461.

18. Cotton, J. P.; Farnoux, B.; Jannink, G. J Chem Phys 1972, 57, 290.

19. Wiltzius, P.; Haller, H. R.; Cannell, D. S.; Schaefer, D. W. Phys Rev Lett 1983, 51, 1183.

20. Koberstein, J. T.; Picot, C.; Benoit, H. Polymer 1985, 26, 673.

21. Brown, W.; Mortensen, K.; Floudas, G. Macromolecules 1992, 25, 6904.

22. King, J. S.; Boyer, W.; Wignall, G. D.; Ullman, R. Macromolecules 1985, 18, 709.

23. Flory, P. J Chem Phys 1949, 17, 303.

24. Brown, W.; Nicolai, T. Colloid Polym Sci 1990, 268, 977.

25. Rapold, R. P.; Suter, U. W.; Theodorou, D. N. Macromol Theory Simul 1994, 3, 19.

26. Destrée, M.; Lyulin, A.; Ryckaert, J.-P. Macromolecules 1996, 29, 1721.

27. Kratky, O.; Porod, G. Recl Trav Chim Pay-Bas 1947, 68, 1105.

28. Jerke, G.; Pedersen, J. S.; Egelhaaf, S. U.; Schurtenberger, P. Phys Rev E 1997, 56, 5772.

29. Jerke, G.; Pedersen, J. S.; Egelhaaf, S. U.; Schurtenberger, P. Langmuir 1998, 14, 6013.

30. Cannavacciuolo, L.; Sommer, C.; Pedersen, J. S.; Schurtenberger, P. Phys Rev E 2000, 62, 5409.

31. Sommer, C.; Pedersen, J. S.; Egelhaaf, S. U.; Cannavacciuolo, L.; Kohlbrecher, J.; Schurtenberger, P. Langmuir 2002, 18, 2495.

32. Cannavacciuolo, L.; Pedersen, J. S.; Schurtenberger, P. J Phys: Condens Matter 2002, 14, 2283.

33. Cannavacciuolo, L.; Pedersen, J. S.; Schurtenberger, P. Langmuir 2002, 18, 2922.

34. Stellman, S. D.; Gans, P. J. Macromolecules 1972, 5,516 .

35. Zimm, B. H. J Chem Phys 1948, 16, 1093.

36. (a) Pedersen, J. S. In Modern Aspects of SmallAngle Scattering; Brumberger, H., Ed.; Kluwer Academic: Boston, 1995; p 57; (b) Pedersen, J. S. In Neutrons, X-Rays and Light; Lindner, P.; Zemb, T., Eds.; Elsevier: Amsterdam, 2002; p 127.

37. Frenkel, D.; Vos, R. J.; de Kruif, C. G.; Vrij, A. J Chem Phys 1984, 84, 4625.

38. Pedersen, J. S. In Neutrons, X-Rays and Light; Lindner, P.; Zemb, T., Eds.; Elsevier: Amsterdam, 2002; p 381. 
39. Aharoni, S. M. Macromolecules 1983, 16, 1722.

40. Rawiso, M.; Duplessix, R.; Picot, C. Macromolecules 1980, 13, 1518.

41. The volume of a chain is taken as the sum of the volume of the spheres, the small overlap of the neighboring spheres being neglected.

42. Calculating the chain number density from the melt density and using the volume of a model chain, we find that the melt corresponds to $\eta$ $=0.154$ for $b=20 \AA$ for chains with six spheres per $b$.

43. Historically, the simulations were performed before the SANS measurements on PS were performed. Although we attempted to choose polymers with molar masses corresponding to the simulation parameters, polymers with exactly corresponding masses were not available.

44. Hansen, J. P.; MacDonald, I. R. Theory of Simple Liquids; Academic: London, 1986.

45. Benoit, H.; Doty, P. J Phys Chem 1953, 57, 958

46. Ohta and Oono ${ }^{2}$ provided a simple expression for the reduced concentration, $X=(16 / 9) \mathrm{CA}_{2} M$ (eq 7), and for $S(0)$. We used these expressions to parameterize the results of Nakanishi and $\mathrm{Ohta}^{3}$ with a more advanced calculation. However, a scale factor, $\epsilon_{4}$, which deviates slightly from unity, is required for the values to agree.
47. We suspect that the crossovers for the different parameters could be system-dependent and depend on chemical details. This is particularly important for the crossover to the high-concentration region (see eqs 14 and 15).

48. Pedersen, J. S. In Neutrons, X-Rays and Light; Lindner, P.; Zemb, T., Eds.; Elsevier: Amsterdam, 2002; p 391.

49. (a) Jacrot, B.; Zaccai, G. Biopolymers 1981, 20, 2413; (b) Lindner, P. In Neutrons, X-Rays and Light; Lindner, P.; Zemb, T., Eds.; Elsevier: Amsterdam, 2002; p 23.

50. Debye, P. J Phys Colloid Chem 1947, 51, 18.

51. Masa, Z.; Porsch, B.; Sundelöf, L. O. Chem Scr 1978, 13, 31.

52. Olsen, B.; Pedersen, J. S. University of Aarhus, Aarhus, Denmark. Unpublished work, 2003.

53. Le Guillou, J. C.; Zinn-Justin, J. Phys Rev B 1980, $21,3976$.

54. Li, B.; Madras, N.; Sokal, A. D. J Stat Phys 1995, 80,661 .

55. Garamus, V. M.; Pedersen, J. S.; Kawasaki, H.; Maeda, H. Langmuir 2000, 16, 6431.

56. Stradner, A.; Glatter, O.; Schurtenberger, P. Langmuir 2000, 16, 5354.

57. The results from refs. 3 and 4 have been scaled in the figures so that the definition $X=(16 / 9) c A_{2} M$ from ref. 2 is used. 